\title{
A Survey of Vehicle Upper-equipment Mounting System Application
}

\author{
Xiaozheng Dang ${ }^{1,2, a}$, Xiaojun Wei ${ }^{1, b}$, Baocheng Ding ${ }^{1, c}$ \\ ${ }^{1}$ Military Transportation Institute, Tianjin, China \\ ${ }^{2}$ School of Mechanical Engineering, Tianjin University, Tianjin, China \\ adangxiaozheng@163.com, ’ weixiaojun@163.com, dingbaocheng@163.com
}

Keywords:. upper-equipment mounting system, application, vibration isolation.

Abstract. Upper-equipment mounting system is playing more and more important role in the field of transportation vibration isolation. This study summarizes configuration categories, development and research status of the mounting system. Research direction future need of the mounting system is also proposed.

\section{Introduction}

Military vehicle upper-equipment mounting system is the assembly structure used to reduce and control the transmission of the vehicle's mounted vibrations and to support it.

At present, the structure type of military vehicle upper-equipment mounting system between the chassis and the upper equipment is rigid. For a long time, the design of these suspension connections is mainly focused on the structural strength, and the design of the suspension structure with the aim of suppressing the vibration transmission is less. Research shows that the vibration of the excitation source without attenuation, is directly transmitted through the frame to the upper equipment, and the deformation of the frame will be caused by the torsional load or the vehicle under the turning and emergency braking conditions. The relative displacement of the upper equipment and the chassis, seriously affects the reliability of the connection structure. The use of unreasonable modified connection structure will worsen the structural stress distribution, cause the resonance phenomenon, and affect the vehicle safety and reliability. In addition, the rigid suspension caused by the installation of high vibration strength, so we have to take vibration isolation measures for the carrier equipment which require higher vibration environment. These will result in a large number of waste space and increased transportation costs.

Based on this, we convert around configuration categories, development and research status of the mounting system, and propose future need of the mounting system.

\section{Theory of suspension damping}

In the theory of suspension damping, a more mature theoretical system has formed. In 1939, Illife[1] proposed the basic principles of the suspension system design. In the 1950s,.Harrison H.C[2] proposed six-degree-of-freedom suspension system decoupling theory and calculation method. In 1979, the design of the system is optimized by Johnson SR to optimize the natural frequency of the system and realize the decoupling of the respective degrees of freedom. In 1990, Demic M.A.[3] optimized the parameters of the suspension system with the response force and response torque at the suspension, and the results were validated by experiments. In 2003, Madjlesi R optimized the vibration isolation performance of vehicle body suspension system based on vibration transfer function. In 2007, Dongfeng Motor Company Zeng Bin[4] conducted a road experiment on a kind of Chinese military vehicle, analyzed the causes of failure of the body suspension structure, and proposed the improvement measures of the suspension system and related parts. In 2011, the finite element method was used to study the suspension system. Based on the finite element method and the modal reduction theory, the stochastic road conditions and the vibration response of the vehicle structure under the pulsed road surface were studied. The cumulative error of the vehicle virtual prototype model was established 
Large, and complex analysis. It is a trend to analyze the development of the suspension model by using the frame - suspension - upper - mounted coupling subsystem combined with the experimental method to obtain the vibration parameters of the loading structure.

\section{Suspension structure design}

In the suspension structure design, the suspension system can be divided into passive, semi-active control and active control according to the suspension system control mode. Because of different control methods, technical difficulty and complexity vary widely. And they apply to the different stages of the vehicle design process. Generally, the performance matching of the semi-active suspension and active suspension in the vehicle chassis design need to be fully considered before they can play its vibration isolation performance advantages. The current research in developed countries is very popular, but because of manufacturing costs, structural size and manufacturing process, reliability and other factors, they has not been widely used. The passive suspension structure is still used widely. Although the passive suspension system is appropriate for a certain frequency range of vibration reduction, but the use of reasonable design, combined with advanced manufacturing technology, can still achieve better damping effect.

The current main passive suspension system consist of multi-point flexible support type, common sub-frame type, and pipe beam fixed type.

Multi-point flexible support system: Through a number of connecting points, the vehicle floor is fixed to the frame by use of the location of the connection of the original chassis. The flexible devices is used at the junction, while the floor at the same time is strengthened. This type is mainly used for smaller tonnage vehicles, as shown in Figure 1.
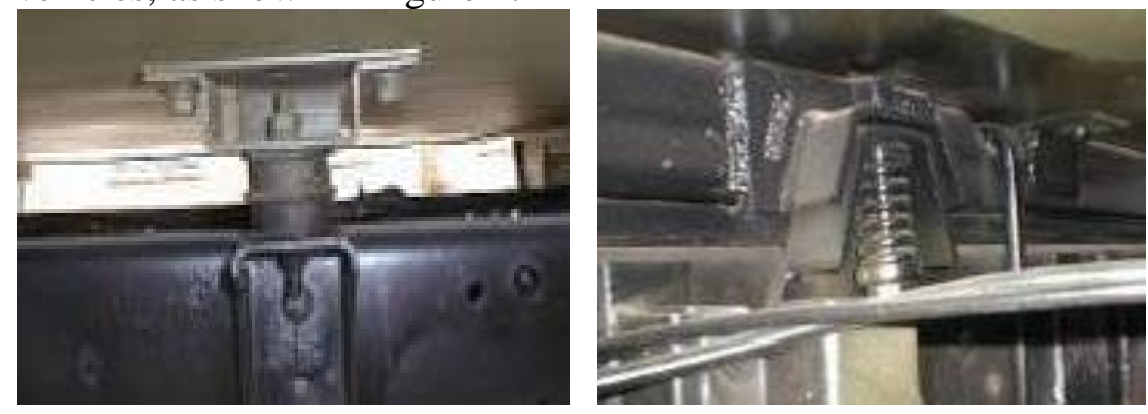

Fig. 1 Multi-point flexible support suspension connection

Figure 2 and Figure 3 describe the connection between the upper equipment platform and the frame of the SCANIA $4 \times 4$ off-road vehicle. The connection take four-point support, diamond-shaped arrangement, the left and right sides of the support with rubber blocks, and before and after two support. The structure don't limit the relative rotation between the vehicle and the frame, thereby attenuating the vibration of the frame to the upper equipment.

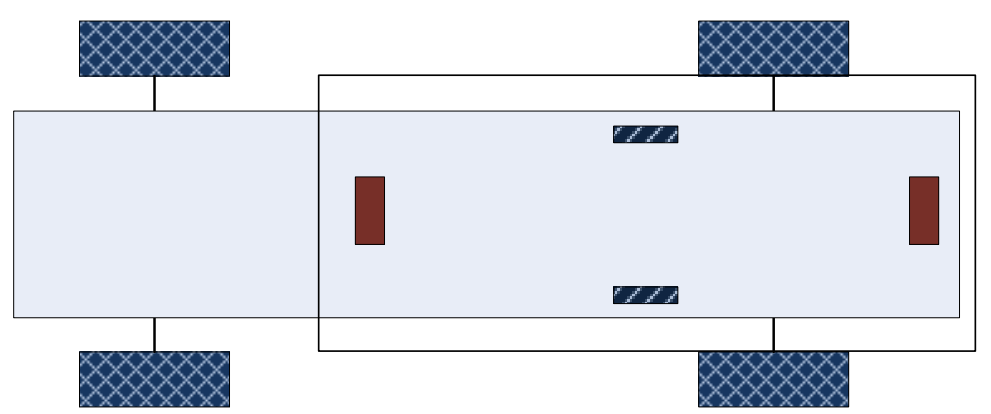

Fig 2 SCANIA $4 \times 4$ off-road vehicle upper equipment and frame four-point support layout 


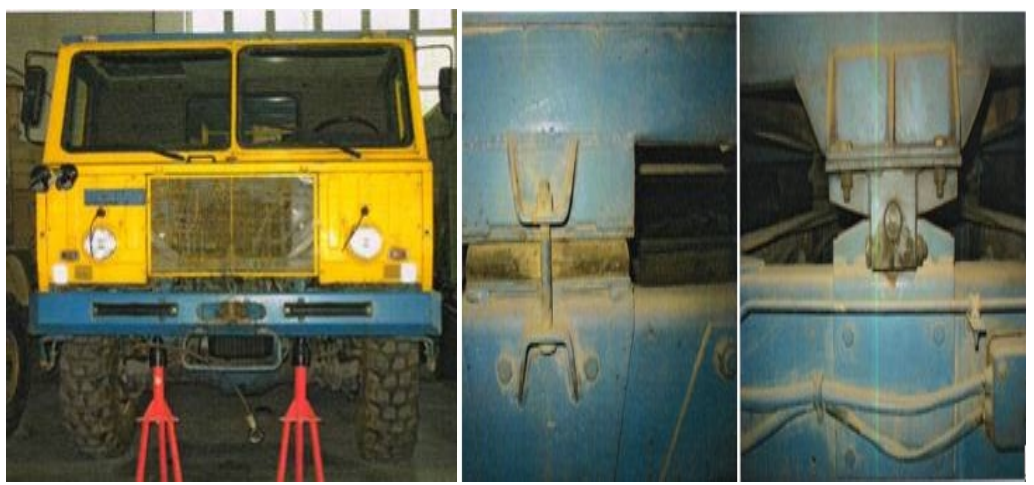

Fig 3 SCANIA $4 \times 4$ off-road vehicle upper equipment and frame connection

EQ2050 $4 \times 4$ off-road vehicle body and frame connection is taken in the form of multi-point rubber support.

Ordinary sub-frame mount system: Ordinary sub-frame mount system is between the upper equipment and the chassis. The upper-equipment bottom and the beams are fixed by bolts. The upper-equipment bottom and sub-frame longitudinal beam are usually elastically connected by U-bolt. This method can improve the support stiffness, and the vehicle during the process of sub-frame can withstand part of the torque. These are conducive to isolation and attenuation of the vibration and shock, widely used in large size of the superstructure.

Pipe beam fixed suspension system: Pipe beam fixed suspension system consists of a pipe beam and some brackets, which are connected to the frame by means of attachment beams. The support structure is to select a number of support points on the beam, which are the smaller deformation point during the various of conditions called the neutral surface support point of the pipe beam, through which the pipe beam, the attachment beam and the frame form a rigid connection. This method can avoid the external torque on the upper-equipment structure directly, improve working conditions and reliability, widely used in medium and heavy military van and extended van.

The pipe beam fixed suspension structure is shown in the BENZ 2026A 6-6 off-road vehicle, as shown in figure 4.
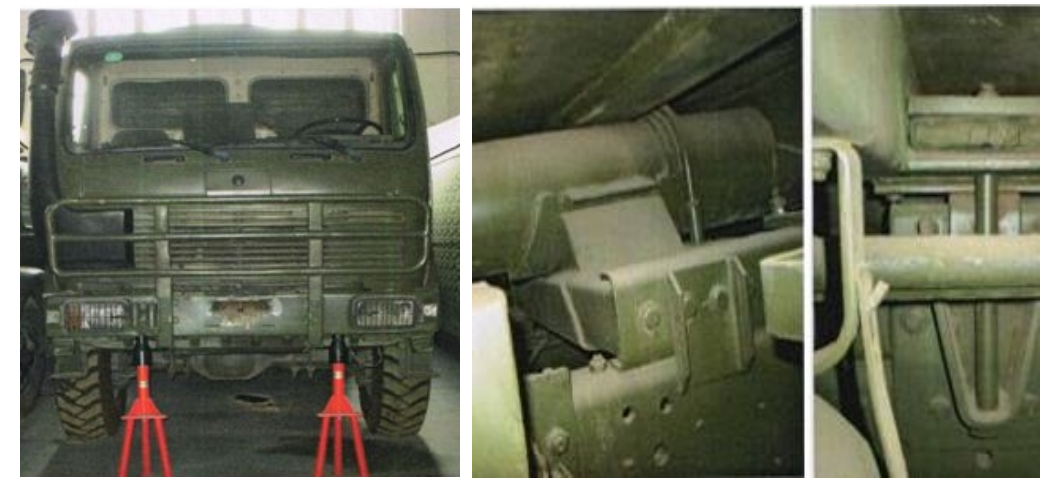

Fig 4 The pipe beam fixed suspension structure in the BENZ 2026A 6-6 off-road vehicle

UNIMOG $30004 \times 4$ off-road vehicles also use pipe-fixed platform. The connection platform by the cross-section of the hexagonal longitudinal beam and equal strength of the beam welded as shown in Figure 5.
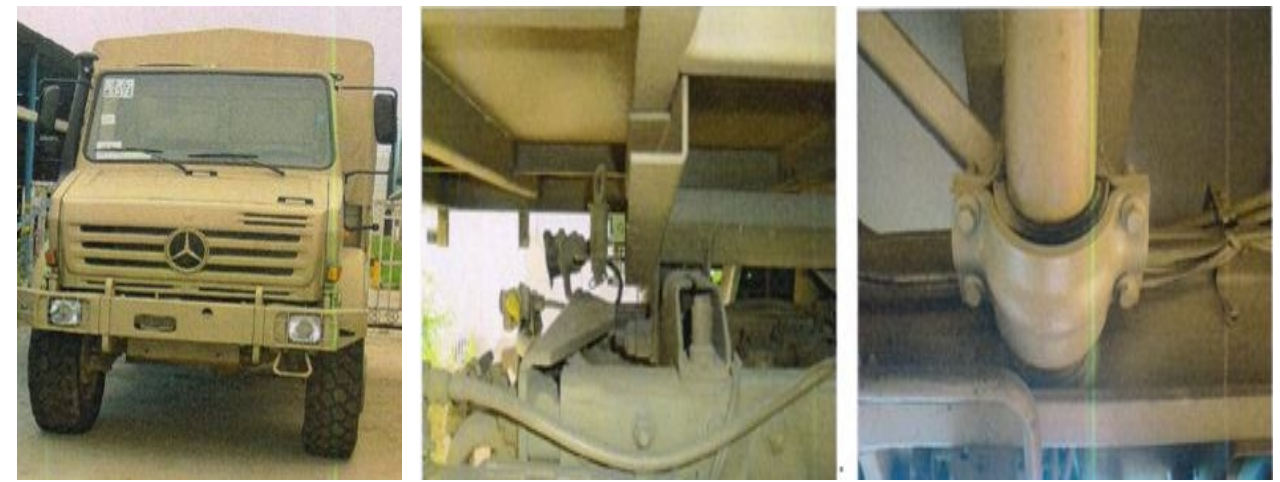

Fig 5 The pipe beam fixed suspension structure in the UNIMOG $30004 \times 4$ off-road vehicle 


\section{Suspension system optimization and matching}

In the 1980s, Johnson[5,6] first applied the optimization technique to the design of the suspension system in order to optimize the natural frequency of the system and realize the decoupling of the vibration between the degrees of freedom. And the suspension arrangement position are optimized for the design variables, so that the vibration coupling between the translational degrees of the system is greatly reduced to ensure that the sixth order natural frequency of the system is within the desired range. Since 1983, Tsinghua University, Xu Shi'an[7], who proposed to minimize the magnitude of the action as the objective function of the suspension, the appropriate control system inherent frequency. 2003 Amir Khajepour took advanced optimization design method in a given vehicle body suspension system design, so that it has the best vibration isolation performance. Around vehicle suspension system parametric modeling, American General Motors Corporation established the relevant parameters of the database system, proposed technical route of the suspension structure optimization, and applied to the actual production, making the suspension system design more efficient.

\section{Conclusions}

Vehicle upper-equipment mounting system is a complicated system. At present, it has formed a complete process of design, development and experiment in the area, and still has a lot of research on the passive suspension system. In the future, it still has very great development space and development potential in the engineering application.

\section{Acknowledgements}

The financial support of the Military Transportation Institute of Tianjin are gratefully acknowledged.

\section{References}

[1] Wan der Walt J.C. On the modal testing of structures with discrete rubber elements. Proceedings of the International Modal Analysis Conference-IMAC, 1991, (1):780-784.

[2] Harrison H.C. Engine installation-a discussion of methods to suit unitary construction. Automobile Engineering, 1956, 46(3):380-383.

[3] Demic M.A. Contribution to the optimization of the position and the characteristics of passenger car powertrain mounts. International Journal of Vehicle Design, 1990, 11(1):87-103.

[4] B. SHEN. The optimal stucture parameter and validation of the mounting system for one kind of military off-road vehicles. Automotive technology. 2008(S1):12-15.

[5] Anon. Reduced shake engine mount system. Automotive Engineering. 1998, 106(4):189-192.

[6] Gordon T.J. On improving the performance of automotive semi-active suspension systems through road preview. Journal of Sound and Vibration. 199, 217(1):163-182.

[7] S.A.XU. Vibration isolation and decoupling technique of engine-mount on vehicles. Automotive Engineering. 1995, 17(4):198-204. 